University of Euroregional Economy in Jozefow,

Poland

\title{
Challenges OF DATING OF FINGERPRINTS AND BLOODSTAINS
}

\begin{abstract}
A reliable dating of forensic traces revealed at the crime scenes is still one of the most challenging issues of forensic science. While there are lot of proprietary research and development methods being promoted and applied within various fields of forensic research, the issue in question remains largely unresolved and as such justifies an evaluation. As fingerprints and bloodstains are nowadays one of the most important types of forensic traces often revealed at crime scenes worldwide and utilized in practice for human identification purposes, a descriptive method has been applied to present current research paths and a potential to provide long awaited resolution of the issue in question. A review of research approaches undertaken by various forensic scientists and joint research teams leads to the conclusion a progress has been achieved through utilization of both new research concepts and application of fast evolving and advancing technologies, yet no scientifically proven ultimate solutions have been presented. This issue remains controversial and still unresolved despite numerous attempts to establish both commonly agreed and reliable markers or characteristics corresponding to a complex issue of ageing of forensic traces, as there are various internal and external factors impacting the process in question.
\end{abstract}

KEYWORDS: forensic traces, age assessment, traces dating, fingerprints, bloodstains.

\section{INTRODUCTION}

Determination of an age of forensic traces, especially biological ones, remains one of the long unresolved major issues of forensic science. Such information would be undoubtedly a great help in any investigation where 
traces have been revealed and would enable provision of a scientific evidence, crucial for determination of any case. It has to be pointed out that assessment of an age of a given trace can in fact enable vital conclusions regarding:

- a timeline of an incident / crime ( when it happened);

- establishment whether examined incident is or is not a crime at all (as the legal regimes evaluate and change over time);

- forensic identification linking given individual with an incident / crime (no matter what actual role played).

Various types of forensic traces are being revealed at crime scenes and among them some are obvious candidates for age assessment - in practice this includes open wounds, bruises, fingerprint latent and bloodstains. Also an ink revealed at the crime scene can be examined to establish an age of handwriting (Bremmer, 2011, p. 15). Among the aforementioned types of traces, fingerprints and bloodstains are of particular interest considering both practical importance in investigations and a share these constitute among all traces revealed nowadays in forensic practice. It has to be also noted revealed bloodstains are often associated with especially serious and violent types of crimes and high-profile cases catching a public attention. Therefore, a lot of effort has been placed by forensic scientific community over a last few decades to develop an acceptable, scientifically proven method of assessment of age of fingerprints and bloodstains with a view to minimize error rates of proposed research methods and ensure admissibility of such evidence at the courts of law.

\section{ISSUES CONCERNING ASSESSMENT OF THE AGE OF FINGERPRINTS}

The 'age of fingerprints' can be defined as the time period from the moment the traces came into existence to the moment they have been revealed and secured. During such time period the traces are exposed to various psychical and chemical phenomena recognized as the actual aging process. In accordance with $\mathrm{K}$. Baniuk this term means the changes that take place both within the sweat and grease substance and on the image of the trace leading to tarnishing, drying and loss of adhesive capabilities (Baniuk, 1981, p. 6). 
These are primarily drying and change of unsaturated adapoid compounds into saturated ones as well as impact of oxygen and steam triggers rancidity of fats, which leads to tarnishing and hardening of natural secretions of perspiratory glands (Lee et al., 2001, p. 87-89).

The speed of aging of the fingerprints depends on various factors including the composition of sweat and grease substance, type of surface where the traces have been left and environmental conditions-temperature, humidity, dusts, air movements and precipitation. The research concerning the aging of fingerprints has not been a topic of interest for Polish experts for quite a long time. Only in 1969 the first research results in the subject matter have been published (Szczepanik, 1969, p. 257-259). This was followed by S. Ośródka, who presented an experiment conducted in the framework of a specific case and formulated conclusions that provided foundation for development of assessment of the age of fingerprint traces. Ośródka claimed 'durability cannot be measured by any particular tool as it is strictly dependent on many, often impossible to capture parameters like type of surface, temperature, humidity, air moisture etc.' ( Ośródka, 1969, p. 624-629). The first scientific publications evaluating impact of humidity on durability of fingerprints have been presented by K. Baniuk and S. Owczarkowski (Baniuk et al., 1970, p. 168-181). From 1975 to 1979 K. Baniuk has conducted research concerning establishment of the age of fingerprints. Approximately 20 thousand of sweat as well as sweat and grease traces have been examined in various environmental conditions, both at open and closed spaces. The research involved smooth and non-absorptive surfaces like glass, metals, and plastic (Baniuk, 1990, p. 133-137).

It was established that tempo of aging of the traces is not steady. Factors that speed this process up are: high temperature (above $30^{\circ} \mathrm{C}$ ), low humidity of air (below 30\%), precipitation, light and contamination of air. Factors slowing it down are: low temperature $\left(0^{\circ} \mathrm{C}\right.$ and below), a lot of grease in the secretions of perspiratory glands, low contamination of air. Similar conclusions have been also confirmed by another research conducted in one of Police forensic laboratories in Poland (Nozdryn-Płotnicki et al., 1995, p. 54-57).

According to the research of $\mathrm{K}$. Baniuk, in the closed space the average lifetime of the trace on glass background was between 3 months to 1,5 years. 
The higher concentration of grease substance, the longer trace lasted. In the open space and different environmental conditions, the average lifetime of trace on glass background varied between 6 and 26 days. On metal background fingerprints lasted shorter - from 3 months to 1 year in closed space and from 4 to 20 days in open space. The shortest was the lifetime of traces on plastic background - from 2 to 7 months in closed space and from 3 to 20 days in open space. The method of the assessment of the age of fingerprint traces developed by K. Baniuk is based solely on psychical characteristics of sweat and grease substance which change over time. The level of such changes can be, in accordance with the author, established through optical means without a need to conduct research on chemical composition of sweat and grease substance. It has to be noted that the aforementioned research provided foundation for development of norms of so-called lifespan of traces, which is an average time of trace existence in different environmental conditions (Baniuk, 2000, p. 63).

More recent experimental approaches to identify a reliable marker of age relevant for fingerprints include utilization of contrast between ridges and valleys in a trace, which is a quantitative variable that may be measured for prints developed with the majority of techniques. Contrast in a print was measured utilizing a method developed by Matuszewski and Szafałowicz (2013), being defined as the difference in the average intensity of pixels between valleys and ridges in a print. Obtained results suggested that the contrast between ridges and valleys in powder-developed prints demonstrates clear changes with the age of a print-however, the pattern of these changes is tied to initial lipid content in examined traces. Traces of high lipid content reveal no change in contrast for a very long time after deposition and therefore contrast between ridges and valleys is practically useless as a marker of age for such traces. As for traces with a low and moderate lipid content, the contrast clearly decreases for a quite long time after the initial period of no-change, which indicates that contrast may be practically useful as a marker of age for traces containing low or moderate amounts of lipids. Therefore, while further extensive research is necessary to address a number of remaining issues, contrast may be at least considered a promising candidate to become a marker of age of fingerprints for at least some categories of these traces (Matuszewski, 2015, p. 5-13). 
It is also worth to mention a comprehensive research in a subject matter conducted in Romania involving 800 fingerprints examined over a period of two years with a view to explore fingerprints aging process and encompassing issues related to:

- ridge thickness,

- distance between ridges and valleys,

- number of macroscopic elements in a trace,

- number of pores,

- number of epithelial cells,

- quantification of total DNA.

Obtained results lead to an interesting suggestion of a possible blood group influence on a fingerprint aging process, involving all four blood groups ( 0 , $A, A B$ and $B$ ). While of course no definitive conclusion is being offered, such concept may provide both interesting and useful information to be utilized in investigations (Popa et al., 2010, p. 149-154).

Issue of assessment of age of the fingerprints is a controversial one, facing criticism from many of the experts. Main problem seems to be the variety of factors impacting the aging process. G.B. George points out that however intriguing the question of age of the fingerprints might be, and irrespectively that all the fingerprint experts would love to be able to make such assessment, the examination of age of traces seems to be fruitless experience (George, 1993, p. 13-16). In turn R. D. Olsen is of the opinion that there are a lot of variable factors impacting the lifecycle of fingerprints and it is impossible to evaluate them, unless full scale comprehensive research is being conducted (Olsen, 1997, p. 117-125).

Other researchers note that the age of the fingerprints cannot be established through technical examination. In case the trace was revealed at non-absorbent background, the only knowledge one can use to assess its age is the date of recent cleaning of the background (Lee et al., 1991, p. 257). Also in Poland there is fundamental discussion regarding reliability of the expert reports regarding assessment of the age of the fingerprints (Marcinkowski, 2000 , p. 58-62). In the current state of knowledge, it still seems impossible to formulate categorical positive conclusions in opinions regarding assessment 
of the age of examined fingerprint traces. Therefore, such conclusions are of probabilistic nature (Baniuk, 2000, p. 63-64).

\section{ISSUES CONCERNING ASSESSMENT OF THE AGE OF BLOODSTAINS (TRACES OF BLOOD)}

Bloodstains are one of the important types of evidence present at crime scenes. They can be used for DNA-profiling or for pattern analysis in order to reconstruct the crime. However, determination of the time elapsed since the crime was committed through assessment of the age of bloodstains is still considered impossible. From a criminalistics point of view, correct estimation when the crime was committed allows to verify witnesses' statements and limits the number of suspects. On one hand it is well understood that this is significant issue for law enforcement institutions, but on the other it is obvious that variety of biological traces and many unpredictable factors impacting aging process make such task extremely difficult.

Forensic experts have been dealing with a challenge of finding methods for determination the age of bloodstains for over a century. The earliest reference in literature is probably by Louis Tomellini from the university of Genoa, Italy in 1907. He developed a chart with 12 figures to illustrate color changes of a bloodstain from the moment of deposition up to one year (Tomellini, 1907, p. 2). In the 1930s, Schwarzacher attempted to correlate the age of a bloodstain with the bloodstain's rate of solubility in water, which is high for fresh bloodstains and then decreases slowly as the bloodstain ages (Schwarzer, 1930, p. 374-380). A few years later Schwarz used a guaiacum-based assay to determine catalase and peroxidase activity of hemoglobin in bloodstains and it showed that the intensity of the reaction's color was inversely related to the age of a bloodstain (Schwarz, 1937, p. 1-34). In the 1950s, Rauschke elaborated on the results of Tommelini and Leers, by analyzing the chemical reaction responsible for the color change of an ageing bloodstain (Bremmer et al., 2012, p. 1-11).

The color of a blood stain changes with time from red to brown which suggests that optical methods can be a considered for age determination. Patterson was the first to recognize this approach in 1960. He recorded blood stain's reflectance spectra and observed that the changing color of the blood 
stain depends on environmental conditions (Patterson, 1960, p. 688-689). In 1972 Patterson, Kind and Owen developed a method of non-destructive bloodstain age assessment using spectrophotometric analysis of visible absorption spectra, which was based on analysis of bloodstains immersed in liquid paraffin (Kind et al., 1972, p. 27-54). Following on this idea, in 1973 Kind and Watson published an alternative method of bloodstains age assessment based on examination of ammoniacal extracts of bloodstains prepared for $\mathrm{ABO}$ grouping by the method of Kind and Cleevely. The measurements are being performed with the light wavelengths of 500, 560, 578 and $650 \mathrm{~nm}$ (Kind et al., 1973, p. 325-332). The later allowed to establish the age of the trace of blood within a period of 10 to 15 years after the formation of the bloodstain. In 1977, Rajamannar proposed utilization of immunoelectrophoresis for determination of the age of traces of blood. The experiment was based on electrophoresis of $1 \mathrm{~cm}$ fibers in agarose gel (put in standard saline) of 15 days to 1 year old bloodstained clothes (Rajamannar, 1977, p. 159-164). The conclusion of research was that this method might be applicable to determine age of traces that are less than 1 year old, due to advancing degradation of Beta and Gamma globulins. Another method proposed by Andrasko utilizes High - Performance Liquid Chromatography (HLPC) for determination of the age of bloodstains existing on clothes (Andrasko, 1997, p. 601-607). The clothes were stored in a closed room of known temperature and humidity for a period of 60 days. It was concluded that HLPC allows to establish the age of the bloodstain provided that environmental conditions of storage (like temperature) are known. In recent years Fujita and others utilized EPR technology to determine age of the blood samples (Fujita et al., 2005 , p. 39-43). Conducted research allowed to establish reliable age of the traces stored in known conditions that are up to 1 year old. This method seems to have two main advantages - it is non - destructive and it requires only $10 \mathrm{mg}$ of the dried blood for examination.

In 2003 Bauer et al published a paper where RNA degradation measurements have been proposed as a candidate for age determination of bloodstains due to the fact that RNA stability is by far lower than DNA. Utilizing fluorescence, it has been demonstrated that quantification of degraded RNA is possible for bloodstains stored up to 15 years (Bauer, 2003, p. 94-103). 
Another approach by Ackerman et al (2010) concentrated on estimation of trace deposition with circadian biomarkers-it generally differs from all other age determination methods as it cannot determine the age of the bloodstain but only the trace disposition time within 24h cycle (Ackerman et al., 2010, p. 387-95).

In 2011, Bremmer et al. proposed diffuse reflectance spectroscopy (DRS) for determination the age of a bloodstain. DRS is based on non-specular reflection of light to determine a material's optical properties. This technique has proven its value in various disciplines ranging from materials science to tissue diagnostics and is routinely used in medicine to determine the oxygen saturation of blood. The approach in question elaborated on recent progress in biomedical optics which allows quantifying the fractions of relevant blood stain chromophores by multi-component spectral fits. Subsequently, these fractions which change with time, are then related to the age of the blood stain. Bremmer et al reconfirmed that age determination of blood stains is feasible under controlled environmental conditions. The excellent short and long-term fits of the DRS measurements in oxy - $\mathrm{Hb}$, met - $\mathrm{Hb}$ and $\mathrm{HC}$ strongly suggest that the oxidation cascade of extracorporeal blood as well as the absorption spectra utilized for these derivatives at a large spectral range are essentially correct. Thus, the combination of oxidation cascade and optical DRS requires further consideration in pursuing age determination of blood stains found under non-laboratory conditions and perhaps even on crime scenes (Bremmer et al., 2011, p. 166-171).

Li et al. (2013) proposed application of visible wavelength hyperspectral image analysis, a non-contact and non-destructive method, to determination of the age of bloodstains up to 30 days old. Their research covered also determination of the age of fresh blood stains (less than one day old). Applied technique predicted the ages of test samples with an overall average error of \pm 1.17 days over the 30 days of measurement, with an increased effectiveness for the first 7 days. It allowed to determine the age of a bloodstain less than seven days old with an error of less than one day in $84 \%$ of test samples. Regarding fresh blood samples proposed method allowed to estimate the age of bloodstain between 0 and $21 \mathrm{~h}$ with an average error of only \pm 0.09 h. It has to be considered a significant development in the subject matter 
and a promising candidate for further studies, taking also into account a low cost experimental setup utilized for research, not requiring high spectral resolution (Li et al., 2013, p. 270-277).

Most recent reports (2017) point to utilization of a modern analytical method based on the combination of Near - infrared Raman spectroscopy (NIR) and advanced statistics to assess an age of bloodstains. Age predictions were performed using partial least squares regression (PLSR) and principal component regression (PCR). Proposed technique utilizing Raman spectroscopy is non-destructive and quick-therefore it can be potentially performed at the crime scene. Actual bloodstain age is claimed to be correctly predicted with $70 \%$ accuracy for up to two years and with ability to distinguish age of trace between hours, days, weeks, months and years (Kyle et al., 2017, p. 1-7).

Over the last two decades, many more techniques have been explored for this forensic challenge. All of these approaches confirm that the physical and chemical properties of blood stains change over time, however up to now no technique has shown a level of certainty required for age determination in forensic practice.

An additional challenge in the age determination is the relative age of one bloodstain compared to another bloodstain, which may have been formed at different time. Unfortunately, little research has been reported in this field. It has to be considered an important issue since order of formation of bloodstains can confirm or exclude specific scenarios, especially in cases of questioned self-defense.

\section{CONCLUSION}

A reliable assessment of forensic biological traces, including but not limited to, fingerprints and traces of blood, is considered by many 'a Holy Grail' of forensic research. While first attempts to provide a resolution have been proposed already almost 90 years ago, there are still no agreed scientifically proven methods and research techniques reaching reliability required for development and introduction of an acceptable trace age prediction standards into a forensic practice.

It has to be however noted that with advancements in technology and evolution of available research means and tools a significant progress has 
been made utilizing a variety of independent approaches. While on one hand a lot of effort is placed into research regarding the subject matter, on the other there are respectable voices in a worldwide forensic community dismissing a quest for trace age assessment as something impossible to accomplish on the grounds of science-due to a variety of unpredictable external and internal factors impacting aging of traces in an unknown, everchanging and often impossible to recreate conditions. Without a doubt, the only thing regarding assessment of an age of forensic traces which remains unchanged, is a controversy surrounding this issue.

Considering potential benefits for both investigations and provision of evidence at the courts of law, as well as an overall progress made, a research regarding subject matter should and undoubtedly will continue worldwide - with a goal to ultimately reach compliance with established criteria of provision of scientific evidence, stipulated in Daubert standard.

\section{References:}

Ackermann K., Ballantyne K.N., Kayser M., Estimating trace deposition time with circadian biomarkers; a prospective and versatile tool for crime scene reconstruction, Int. J. Legal Med. (2010), ISSN: 1437-1596, p. 387-95

Andrasko J., The estimation of age of bloodstains by HPLC analysis, J. Forensic Sci, July 1997, 42 (4), ISSN: 0022-1198, p. 601-607.

Baniuk K. Kryminalistyczna problematyka oceny wieku śladów linii papilarnych (ślady w pomieszczeniu zamkniętym), Część I, Zeszyty Metodyczne KGMO 1981, nr 27, p. 6.

Baniuk K., Determination of age of fingerprints, Forensic Science International, 46 (1990), ISSN: 0379-0738, p. 133-137.

Baniuk K., Owczarkowski S., Wilgoć a ślady daktyloskopijne, Problemy Kryminalistyki 1970, nr 84, ISSN: 0552-2153, p. 168-181;

Baniuk K., Znaczenie eksperymentu laboratoryjnego w ekspertyzie wieku śladów linii papilarnych, Problemy Kryminalistyki, 2000, ISSN: 0552-2153, 228, p. 63-64;

Bauer M., Polzin S., Patzelt D, Quantification of RNA degradation by semi-quantitative duplex and competitive RT-PCR: a possible indicator of the age of bloodstains? Forensic Science International 138(2003), ISSN: 0379-0738, p. 94-103

Bremmer R.H., Non-contact spectroscopic age determination of bloodstains, University of Amsterdam, 2011, ISBN: 9789490371975, p. 15 
Bremmer R.H., de Bruin K.G., van Gemert M.J.C., van Leeuwen T.G., Aalders M.C.G., Forensic quest for age determination of bloodstains, Forensic Science International 2012, no 216, ISSN: 0379-0738, p. 1-11

Bremmer R.H., Nadort A., van Leeuwen T.G., van Gemert M.J.C., Aalders M.C.G., Age estimation of blood stains by hemoglobin derivative determination using reflectance spectroscopy, Forensic Science International 206 (2011), ISSN: 0379-0738, p. $166-171$ 
